\title{
Variation of Soil Bacterial Communities during Lettuce Continuous Cropping
}

\author{
Jie Hong ${ }^{1}$, Yue Yang ${ }^{1 *}$, Yi Gao ${ }^{1}$, LianQuan Zhong ${ }^{2}$, QuanMing $\mathrm{Xu}^{2}$, XinXin Yi ${ }^{1}$, YiQian Liu ${ }^{1 \#}$ and XiuZhi Gao ${ }^{1 \#}$ \\ ${ }^{1}$ Beijing Laboratory of Food Quality and Safety, Beijing Key Laboratory of Agricultural Product Detection and Control of Spoilage \\ Organisms and Pesticide Residue, Beijing Engineering Laboratory of Probiotics Key Technology Development, Faculty of Food \\ Science and Engineering, Beijing University of Agriculture, Beijing 102206, China. \\ ${ }^{2}$ Beijing Changping District Seed Management Station, Beijing 102200,China. \\ * These authors contributed equally to this work.
}

\begin{abstract}
The variation of bacterial community in lettuce continuous cropping was determined by high throughput sequencing. During the continuous planting of lettuce, the richness and diversity of bacterial communities in the soil increased, and the ACE index and Chao index increased by $40.21 \%$ and $36.91 \%$, respectively. The proportion of Actinobacteria, Chloroflexi, Firmicutes and Nitrospirae in the soil increased, while the abundance of Acidobacteria, Bacteroidetes, Gemmatimonadetes, Planctomycetes and Proteobacteria gradually declined. And the abundance in the soil accounting for $1 \%$ of the dominant bacterial genera increased to 11, among them, Anaerolinea, Bacillus, Nitrosomonas, and Xanthomonas etc became the dominant bacterium genus in the soil after lettuce continuous cropping. After the lettuce had been planted 8 times, the yield decreased by $21.20 \%$ compared to the first harvest. Lettuce continuous cropping had an effect on bacterial community and lettuce yield to some extent.
\end{abstract}

\section{Introduction}

Lettuce (Lactuca sativa L.) is an annual herb of the family Asteraceae, which in vitamins, carotenoids, fiber, as well as polyphenols. Lettuce contains a variety of minerals and beneficial to human health, such as Fe, Zn, $\mathrm{Ca}, \mathrm{P}, \mathrm{Mg}, \mathrm{Mn}, \mathrm{K}$, etc. Lettuce has been eaten by more and more people as a healthy food in recent years in China $^{[1-2]}$. Lettuce can be divided into six types, namely crisphead lettuce, butterhead lettuce, romaine or cos lettuce, leaf or cutting lettuce, steam or stalk lettuce and latin lettuce, according to the shape, size, texture, head formation and stem type of lettuce leaves ${ }^{[3]}$. Spain, Italy and France are the main lettuce producing countries in Europe, with production accounting for $35 \%, 21 \%$ and $13 \%$ of total lettuce production in Europe, respectively ${ }^{[4]}$. China is one of the important country in the output of lettuce inthe world ${ }^{[5]}$. In 2011-2016, only the proportion of leafy vegetable plantings in Beijing accounted for $44.0 \%$ of total vegetable acreage ${ }^{[6]}$. According to survey, in 2013 , the area planted with leafy vegetables in Beijing was 30000 hectares, of which lettuce accounted for $14.4 \%$ and the output reached 200 million kilograms ${ }^{[7-8]}$. Due to the planting area is finite, the demand of lettuce rises to be able to bring about continuous cropping obstacle, unavoidably. Long-term cultivation of lettuce may be susceptible to disease, such as downy mildew, gray mold and virus disease, as described by $\mathrm{Cui}^{[9]}$. Because of the continuous cropping, some hazardous substance were accumulated in the soil, which became the cause of the disease in the following year. Microor-ganisms are efficient bioindicators of soil biological characteristics, because their ability to respond quickly to environmental changes ${ }^{[10]}$. Many studies had shown that changes in the size and activity of the soil microbial community were major contributors to soil degradation caused by agricultural management ${ }^{[11]}$. Soil organisms play important roles in maintaining soil health and quality ${ }^{[12]}$. The purpose of this study is to analyze the variation of soil bacterial communities during lettuce continuous cropping for eight times.

\section{Materials and Methods}

\subsection{Field experiment}

The experimental field is located in the plastic greenhouse of the crop variety test demonstration base (116.14 ${ }^{\circ}$ east longitude, $40.19^{\circ}$ north latitude) in Changping District, Beijing. The test field is $400 \mathrm{~m}^{2}(50$ $\mathrm{m}$ long, $8 \mathrm{~m}$ wide), soil type is sandy loam. The planting density is 80 plants per bed, and the plant spacing is 0.3 $\mathrm{m} \times 0.3 \mathrm{~m}$. Duringthe experiment, organic fertilizer was applied at $3000 \mathrm{~kg} / \mathrm{acre}$, and the basic physical and chemical indicators of the soil were tested: soil alkali $\mathrm{N}$ $153.21 \mathrm{~g} / \mathrm{kg}$, soil available P $270 \mathrm{~g} / \mathrm{kg}$, soil available K $312.45 \mathrm{~g} / \mathrm{kg}, \mathrm{pH}$ 6.90. The planting lettuce variety is North Sansheng No. 2. The test wasconducted from September 2016 to June 2018. The specific sample

\footnotetext{
\# Corresponding author: gxz@bua.edu.cn;
}

liuyi143@163.com 
information is shown in Table 2-1. The continuous crop has 12 beds, $6.5 \mathrm{~m}$ long and $1.2 \mathrm{~m}$ wide, in winter had a leisure period. The sample naming method is N_planting year_ planting times_planting (1)/ harvest (2),for example: N_17_5_1 indicates the 5th lettuce in 2017 before planting.

According to the experimental design, thesampling range is determined, the surface floating soil is removed, the soil with a depth of $0-20 \mathrm{~cm}$ is excavated, and the visible impurities are removed. After the sample is collected, it is loaded into the sampling bag and numbered.Each sample was sampled by 5 -point sampling method. After mixing and removing impurities, 5-10 g was taken, stored in a sterile centrifuge tube, placed in an incubator, and transport-ed to the laboratory in 1 hand store at $-80{ }^{\circ} \mathrm{C}$.

\subsection{Determination of lettuce yield}

When the lettuce was harvested, fresh plants were gathered, and 9 plants were randomly taken from each bed and repeated 3 times to determine the fresh weight of the plants.

Table 1. Description of soil samples

\begin{tabular}{ccccc}
\hline Samples & Collection date & Depth (cm) & State of crop growth Cultivation time \\
\hline CK & 2016.09 .09 & $0-20$ & - & - \\
\hline N_16_1_1 & 2016.09 .09 & $0-20$ & Before cultivation & 1st \\
\hline N_16_1_2 & 2016.10 .20 & $0-20$ & Harvest & 1st \\
\hline N_17_2_1 & 2017.03 .10 & $0-20$ & Before cultivation & 2nd \\
\hline N_17_2_2 & 2017.04 .27 & $0-20$ & Harvest & 2nd \\
\hline N_17_3_1 & 2017.05 .23 & $0-20$ & Before cultivation & 3rd \\
\hline N_17_3_2 & 2017.06 .20 & $0-20$ & Harvest & 3rd \\
\hline N_17_4_1 & 2017.07 .02 & $0-20$ & Before cultivation & 4ht \\
\hline N_17_4_2 & 2017.07 .26 & $0-20$ & Harvest & 4th \\
\hline N_17_5_1 & 2017.09 .19 & $0-20$ & Before cultivation & 5th \\
\hline N_17_5_2 & 2017.10 .20 & $0-20$ & Harvest & 5th \\
\hline N_17_6_1 & 2017.11 .05 & $0-20$ & Before cultivation & 6th \\
\hline N_17_6_2 & 2017.12 .13 & $0-20$ & Harvest & 6th \\
\hline N_18_7_1 & 2018.03 .20 & $0-20$ & Before cultivation & 7th \\
\hline N_18_7_2 & 2018.05 .03 & $0-20$ & Harvest & 7th \\
\hline N_18_8_1 & 2018.05 .18 & $0-20$ & Before cultivation & 8th \\
\hline N_18_8_2 & $2018.06 . .21$ & $0-20$ & Harvest & 8th
\end{tabular}

\subsection{Total DNA extraction and amplification of $16 S$ rRNA}

Microbial DNA was extracted from $1.0 \mathrm{~g}$ soil samples using the E.Z.N.A.® soil DNA Kit (Omega Bio-tek, Norcross, GA, U.S.) according to manufacturer's protocols. The quality of the extracted DNA was determined using agarose gel electrophoresis $(0.8 \%)$, and the DNA was quantified using a UV spectroph-otometer. The extracted DNA was stored at $-80^{\circ} \mathrm{C}$ prior to analysis. The V3-V4 region of the bacteria $16 \mathrm{~S}$ ribosomal RNA gene were amplified by PCR $95^{\circ} \mathrm{C}$ for $3 \mathrm{~min}$, followed by 25 cycles at $95^{\circ} \mathrm{C}$ for $30 \mathrm{~s}, 50^{\circ} \mathrm{C}$ for $30 \mathrm{~s}$, and $72^{\circ} \mathrm{C}$ for $45 \mathrm{~s}$ and a final extension at $72^{\circ} \mathrm{C}$ for $10 \mathrm{~min}$ using primers 338F(5'-barcode-ACTCCTACGGGAGGCAGCAG)-3'a nd 806R(5'-GGACTACHVGGGTWTCTAAT -3')[13], according to previously published protocols, where barcode is an eight-base sequence unique to each sample. PCR reactions were performed in triplicate $20 \mu \mathrm{L}$ mixture containing $4 \mu \mathrm{L}$ of $5 \times$ FastPfu Buffer, $2 \mu \mathrm{L}$ of 2.5 $\mathrm{mM}$ dNTPs, $0.8 \mu \mathrm{L}$ of each primer $(5 \mu \mathrm{M}), 0.4 \mu \mathrm{L}$ of FastPfu Polymerase, and 10 ng of template DNA.

\subsection{Illumina MiSeq sequencing}

Amplicons were extracted from $2 \%$ agarose gels and purified using the AxyPrep DNA Gel Extraction Kit (Axygen Biosciences, Union City, CA,U.S.) according to the manufacturer's instructions and quantified using QuantiFluor $^{\mathrm{TM}}$-ST (Promega,U.S.). Purified amplicons were pooled in equimolar and paired-end sequenced $(2 \times 250)$ on an Illumina MiSeq platform according to the standard protocols.

\subsection{Processing of sequencing data}

Raw fastq files were demultiplexed, quality-filtered using QIIME (version 1.17) with the following criteria: (i) The $300 \mathrm{bp}$ reads were truncated at any site receiving an average quality score $<20$ over a $50 \mathrm{bp}$ sliding window, discarding the truncated reads that were shorter than 50 bp. (ii) exact barcode matching, 2 nucleotide mismatch in primer matching, reads containing ambiguous characters were removed. (iii) only sequences that overlap longer than $10 \mathrm{bp}$ were assembled according to their overlap sequence. Reads which could not be assembled were discarded. Operational Units (OTUs) were clustered with $97 \%$ similarity cutoff using UPARSE(ver-sion7.1http://drive5.com/uparse/)and chimeric sequences were identified and removed using UCHIME. The taxonomy of each 16S rRNA gene sequence was analyzed by RDP Classifier (http://rdp. cme.msu.edu/) againstthe silva (SSU115) 16S rRNA database using confidence threshold of $70 \%{ }^{[14]}$

\section{Results}

As shown in Fig.1, the yield of lettuce in the previous four plantings was $5.14 \mathrm{~kg} / \mathrm{m}^{2}, 5.29 \mathrm{~kg} / \mathrm{m}^{2}, 5.01 \mathrm{~kg} / \mathrm{m}^{2}$ and $4.96 \mathrm{~kg} / \mathrm{m}^{2}$, respectively, and the total remained at about $5.10 \mathrm{~kg} / \mathrm{m}^{2}$. With the increased of continuous cropping times, the yield of lettuce decreased significantly during the 5th harvest, which was $13.96 \%$ lower than that of the 1 st harvest. Then, the yield decreased by $16.24 \%, 16.04 \%$ and $21.20 \%$ respectively, in 6th, 7th and 8th planting times. This indicates that as the time of continuous crops increases, it will have an impact on the yield of lettuce, and the yield will decrease significantly after5th lettuce continuous croppings.

The rarefaction curve reflects the depth of sampling and can be used to assess whether or not the number of sequences is sufficient to cover all taxa. The bacteriarichness rarefaction curve showed that, as the number of sequences increases, the increase 


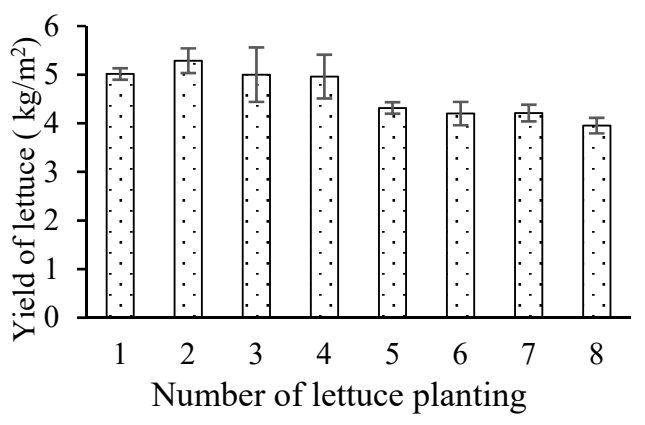

Fig.1. Lettuce yield during continuous cropping

in the number of OTUs in each sample tends to flatten and eventually reaches saturation. The amount of coverage obtained during the sample sequencing was calculated at a similarity level of $97 \%$. The results showed that the amount of coverage in all of the sample libraries was adequate. The rate was approximately $99 \%$, indicating that the sampling was sufficient and the confidence in the structure of the bacteria community that was obtained in the real environment was high, which indicates that our analysis truly reflects the bacteria community present in the soil samples(Fig. 2).

\subsection{Soil bacterial diversity and community str ucture during continuous cropping}

The bacterial 16S rRNA diversity index in continuous cropping soil samples was analyzed (Table 2), and the bacterial diversity index fluctuated with the increased of continuous cropping times. The bacterial diversity index of continuous cropping soil samples showed an increasing trend except shannon index, which decreased by $0.46 \%$. OTU increased from 2010 to 2623, which increased by $30.50 \%$. ACE index, Chao index and Simpson index increased by $40.21 \%, 36.91 \%$ and $26.55 \%$. This indicated that continuous cropping could increase the diversity of bacteria in soil.As shown in Fig. 3 , before continuous cropping of lettuce, the dominant bacterial phyla with more

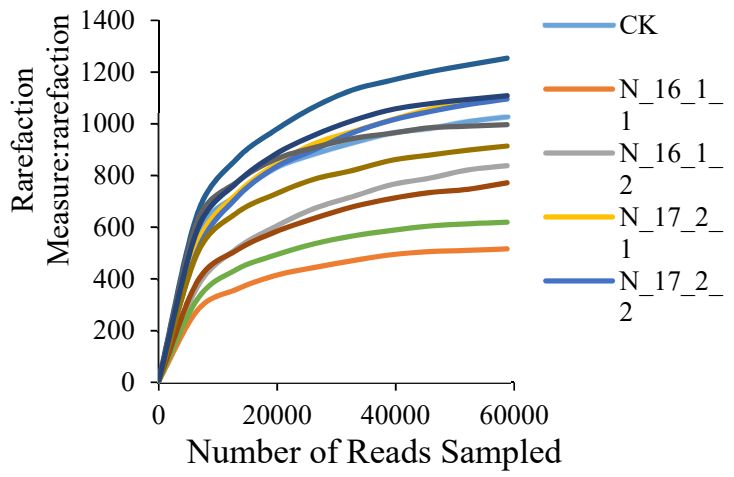

Fig.2. Rarefaction curves indicating the OTUs observed at a genetic distance of $3 \%$ in all soil samples.

than $5 \%$ abundance in the soil was Proteobacteria, Acidobacteria, Bacteroidetes, Chloroflexi, Gemmatimonadetes and Actinobacteria, accounting for $41.36 \%, 21.23 \%, 8.01 \%, 7.75 \%, 6.57 \%$ and $5.72 \%$ respectively in the soil, accounting for $90.65 \%$ in total. Firmicutes, Nitrospirae and Planctomycetes, which account for $1-5 \%$ of abundance, account for

Table 2 Index of bacterial richness and diversity inlettuce continuous cropping soil samples

\begin{tabular}{|c|c|c|c|c|c|c|}
\hline Samples & OTU & $\mathrm{ACE}$ & Chao & Simpson & Shannon & Coverage \\
\hline $\mathrm{CK}$ & 2010 & 2196.3776 & 2238.4194 & 0.0029 & 6.5981 & 0.9934 \\
\hline $\mathrm{N} \_16 \ldots 1$ & 2330 & 2461.4484 & 2519.1346 & 0.0046 & 6.5293 & 0.9940 \\
\hline $\mathrm{N} \_1612$ & 2267 & 2439.0364 & 2490.7783 & 0.0048 & 6.5307 & 0.9931 \\
\hline $\mathrm{N} \_17221$ & 2372 & 2502.4326 & 2544.6884 & 0.0033 & 6.7137 & 0.9942 \\
\hline $\mathrm{N}_{-} 1722$ & 2440 & 2545.4091 & 2561.0041 & 0.0035 & 6.7126 & 0.9947 \\
\hline $\mathrm{N} 17{ }_{-} 3 \mathrm{l}$ & 2399 & 2501.7245 & 2536.5756 & 0.0026 & 6.8160 & 0.9947 \\
\hline $\mathrm{N}_{-} 17332$ & 2469 & 2538.7585 & 2542.3797 & 0.0026 & 6.8606 & 0.9958 \\
\hline $\mathrm{N} \_1751$ & 2793 & 3219.1124 & 3239.8060 & 0.0039 & 6.6393 & 0.9875 \\
\hline $\mathrm{N} 1752$ & 2727 & 3117.2521 & 3119.2598 & 0.0037 & 6.6618 & 0.9883 \\
\hline $\mathrm{N} \_1881$ & 2465 & 3026.7693 & 3086.6196 & 0.0057 & 6.3946 & 0.9868 \\
\hline $\mathrm{N} 1882$ & 2623 & 3079.4499 & 3064.7124 & 0.0037 & 6.5678 & 0.9875 \\
\hline
\end{tabular}

$2.86 \%, 1.54 \%$ and $1.70 \%$, respectively, in soils. As the growth of the continuous cropping time, dominant bacterial in the soil phyla proportion also gradually changed, Actinobacteria, Chloroflexi, Firmi-cutes and Nitrospirae the proportion in the soil was increasing after lettuce planting for 8 times, account-ed for $13.88 \%$, $19.48 \%, 14.16 \%$ and $2.58 \%$ res-pectively, compared with before the continuous cropping were increased by $8.16 \%, 11.73 \%, 11.31 \%$ and 1.04\%. However, Acidobacteria, Bacteroidetes, Gemmatimonadetes, Planctomycetes and Proteobacteria, abundance accounting in the soil decreased, the relative abundance in soil was $18.66 \%, 2.72 \%, 2.01 \%, 1.52 \%$ and $22.13 \%$ respectively, which decreased by $2.57 \%$, $5.29 \%, 4.56 \%, 0.18 \%$ and $19.23 \%$. It can be seen that continuous cropping has a certain impact on the composition of soil bact-erial community.

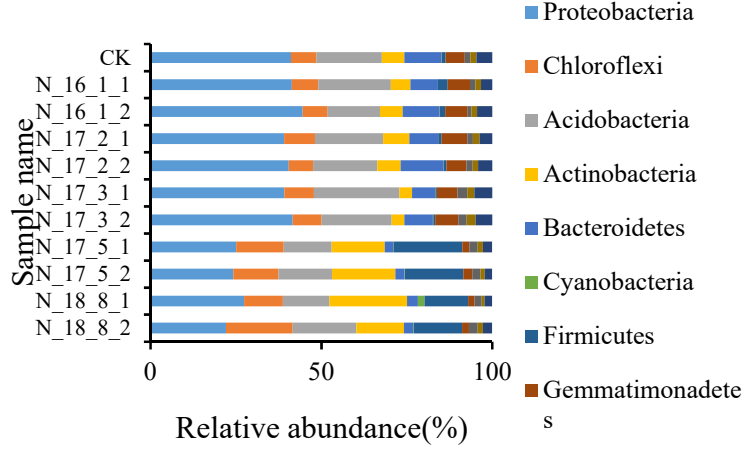

Fig. 3. Relative abundance of the bacterial phyla presents in the continuous cropping soil samples.

There are 8 dominant bacteria genus belonging to the soil with a relative abundance of more than $1 \%$ at the 
beginning of planting, as shown in Fig.4, Adhaeribacter, Bacillus, Bryobacter, H16, Lysobacter, Pontibacter, RB41 and Sphingomonas, the proportion was $1.06 \%, 1.84 \%$, $1.31 \%, 1.89 \%, 6.22 \%, 1.10 \%, 2.10 \%$ and $7.29 \%$, respectively. After 8 times lettuce cropping, soil dominant genus were Bacillus, H16, Nitrospira, norank_c_Acidobacteria, norank_c_KD4-96, norank $\mathrm{f}$ Anaerolineaceae, norank_f_Gemmatimonadaceae,

norank_f_Nitrosomonadaceae, norank_o_AKYG1722, norank o JG30-KF-CM45

andnorank o Xanthomonadales, accounting abundance in the soil were was $7.32 \%, 1.21 \%, 2.58 \%, 15.52 \%$, $2.18 \%, 6.70 \%, 1.19 \%, 1.19 \%, 1.09 \%, 4.04 \%, 1.37 \%$, the type and number of advantagescompared with the previous species planted have un-dergone significant. The change of dominant bacteriafrom 8 to 11, of which only Bacillus and H16 is the dominant genus in the soil through the lettuce con-tinuous cropping. The results showed that the contin-uous cropping of lettuce can increase the diversity of bacterial communities in the soil, and the compoi-tionof bacterial communities in the soil can be changed with the increased of continuous cropping time.

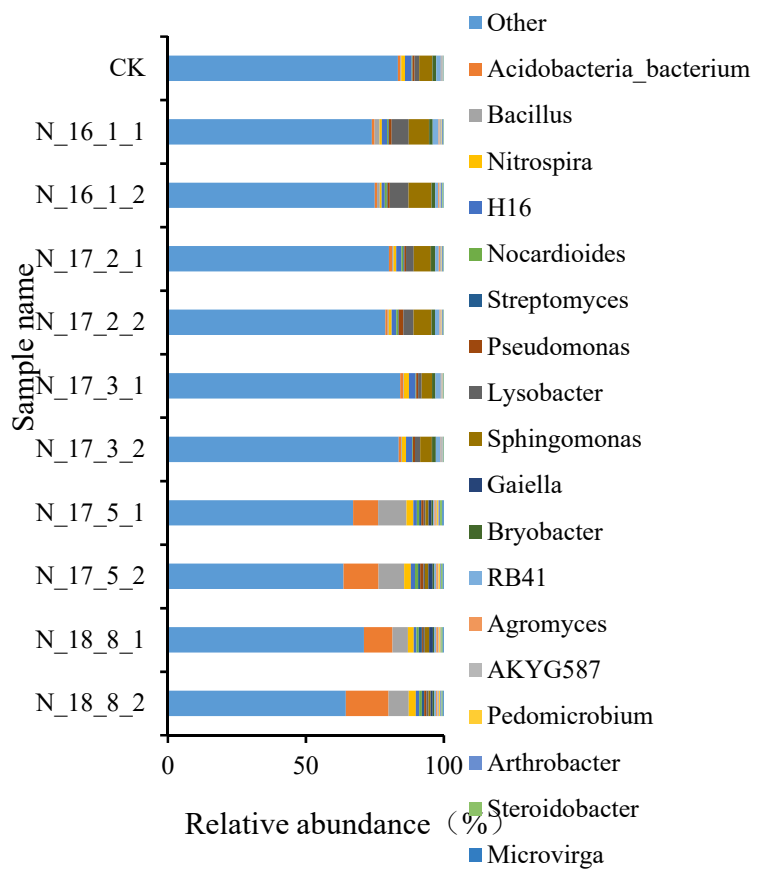

Fig. 4. Relative abundance of the bacterial genus presents in the continuous cropping soil samples

At the genus level, clusters of the genus of the sample and the sample are clustered. According to the different OTU numbers in each sample after clustering, a Heatmap is generated corresponding to the abundance of the contained sequence (Fig.5), and the change of the color gradient can reflect thedifference in bacterial community structure of each sample at the level of the genus. The abundance ofthe genus is affected by the conditions of lettuce co-ntinuous cropping, norank_o_Anaerolineaceae, nor-ank_c_Acidobacteria, Bacillus, norank_c_KD4-96, nor-ank_o_JG30-KF-CM45 and Nitrospira.

\section{Discussion}

From the results of high-throughput sequencing, the coverage of each sample in continuous soil was over $99 \%$, which proved that the sequencing results can represent the real situation of microorganisms in the soil. With the continuous cropping of lettuce, the diversity and richness of bacterial communities in the soil increased gradually, and the ACE index and Chao index increased by $40.21 \%$ and $36.91 \%$,respectively. The higher is the diversity index, the higher the diversity of microbial communities in the soil, which is mainly composed of the diversity and richness of the community ${ }^{[15]}$. Zhao et al. foundthat thespecies richness of bacterial communities in soil increased after 1 year of continuous crop-ping, which was consistent with our experiment, but afterstrawberry continuous cropping for 8 years, the species richness ofsoil bacterial communities is reduced,which is different fromour experiment result. It may be due to the short planting period of lettuce, and the diversity of bacteria has not decreased significantly ${ }^{[16]}$.

The continuous cropping of lettuce also has an effect on the composition of the bacterial community in the soil. During the continuous cropping of lettuce, the species of dominant bacteria in the soil did not change were Proteobacteria, Acidobacteria, Bacteroides, Chloroflexi, Gemmatimonadetes, Actinob-acteria, Firmicutes, Nitrospirae and Planctomycetes, but their relative abundance changes in the soil, inc-luding Actinobacteria, Chloroflexi, Firmicutes and Ni-trospira in the soil the proportion isincreasing, and Acidobacteria, Bacteroides, Gemmatimonadetes, Planc-tomycetes and Proteobacteria, gradually decreased inthe soil. This is consistent with the results of Ouyang et al. who found that the abundance of Firmicu-tes, Actinomycetes and Chloroflexi increased in the soil of banana continuous cropping for 5 years, and became the main bacterial population in the soil[17]. Zhao et al. studies had shown that the abundance of Proteobacteria and Bacteroides increasing can pro-mote the transformation of organic matter in soil, which may had an impact on crop yields, while in this experiment both decline in the process of lettu-ce continuous cropping and the lettuce yield fellby $21.20 \%$ after 8 th lettuce continuous cropping[18].

After 8 times continuous crops of lettuce, the abundance of Anaerolineaceae in the soilincreased, becoming the dominant genus in the soil. Anaerolineaceae is a representative group of the Chloroflexi which is a kind of facultative anaerobic organism andcan not produce oxygen in photosynthesis and can not nitrogen fixation, but it can decompose organic matter and degrade in anaerobic environment[19-20]. The abundance of Anaerolineaceae increase during continuous cropping may affect the absorption and utilization of nitrogen by plants. In addition, Bacill- 


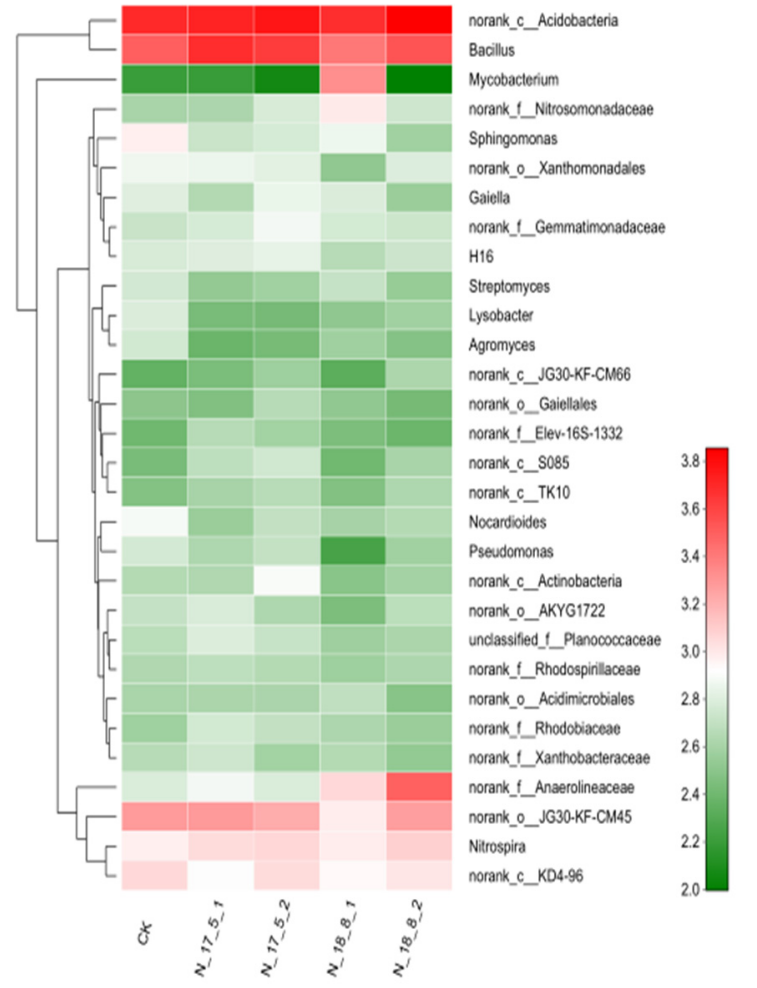

Fig. 5 Heatmap of the bacterial genus present of the cont-nuous cropping soil samples

-us, Nitrosomonas, and Xanthomonas are the domin-ant genus in the soil after 5 years of continuous cul-tivation of Artemisia annua L, and these three genu-s also become the dominant genus in the soil aftercontinuous lettuce production[21]. Studies had found that Sphingomonas can absorb $\mathrm{Cd}$ in soil and degrade a variety of aromatic chemical contaminants[22]. The abundance of Sphingomonas in the continuous soil of lettuce also decreased from $7.29 \%$ to $0.77 \%$, which may affect the degradation of $\mathrm{Cd}$ and aromatic chemical pollutants in the soil, resulting in accumulation in the soil, endangering the growth of lettuce plants.

From the heatmap and community structure, the diversity and abundance of bacteria do not change obviously, which is the same with most field conclusion. Through the observation of potato continuous cropping for 7 years, the abundance of Fusarium int-he soil increased, and the soil-borne diseases became more serious, which in turn affected crop growth ${ }^{[23]}$. Continuous cropping had inhibitory effectson bacteria and nitrogen fixation bacteria, but promote the growth and reproduction of fungi. The soil is converted from bacterial to fungal, from high fertility to low fertility ${ }^{[24-25]}$. After the continuous cropping, the nematode communities of the dominant genus such as the bacterial nematode and the fungus nematode were found to be significantly reduced, while the nematodes with the dominant species of plant parasitic nematodes began to destroy the host plants, which made the soil unfavorable for crop growth, which led to reduced yield ${ }^{[26]}$. The results showed that the main influencing factors of crop continuous cropping are fungal and nematode diseases, while the number of bacteria will gradually decrease, and fungal diseases will be serious year by year, which is also consistent with the trend of smaller changes in the abundance of bacteria in heatmap in this experiment. The results showed that variation of bacterial community in soil of short continuous cropping was not the main factor affecting lettuce yield.

\section{Conclusion}

In this experiment, the diversity and richness of bacterial community in the soil of lettuce continuous cropping increased, but their relative abundance changed in the soil. Their relative abundance altered in the soil, including Actinobacteria, Chloroflexi, Firmicutes and Nitrospirae,while the proportion increased. Other abundance:Acidobacteria,Bacteroides, Gemmatimonadete s, Planctomycetes and Proteobacteria was gradually decreased in the soil. In addition, Bacillus, Nitrosomonas and Xanthomonas became the dominant genus in the soil after continuous lettuce production. From the heatmap and community structure,the diversity and abundance of bacteria did not alter obviously. The change of bacterial community in short continuous cropping soil was not the main factor affecting lettuce yield.

\section{Acknowledgements}

This work was supported by the Beijing Leafy Vegetables Innovation Team of Modern Agro-industry Technology Research System (BAIC07-2019).

\section{Reference}

1. G Y. Luo. High quality lettuce planting technology and econ-omic benefits. Jilin Vegetable,5, 10-10 (2015)

2. M J. Kim , Y. Moon and D A. Kopsell, et al. Nutrit-ional value of crisphead 'iceberg' and romaine lettuces (La-ctuca sativa L.). J Agr Sci-Cambridge, 8(11) (2016)

3. E. Křístková ,I. Doležalová and A. Lebeda,et al. Description of morphological characters of lettuce (Lactuca sati-va L.) geneti-c resources. Hortic Sci, 35(3), 113-129 (2008)

4. B. Virginie, L. Franois and N. Philippe, et al. Lettuce cropping with less pesticides. review. Agron. Sustainable Dev, 34(1), 175-198 (2014)

5. K. Moo Jung ;Y. Moon and T J. C, et al. Nutritionalvalue, bioactive compounds and health benefits of lettuce (Lactuca sativ-a L.). J Food Compos Anal, 49, 19-34 (2016)

6. B. Zhang, N. Xu and L X . Zhang. Analysis of current sit-uation and changing trend of leaf vegetable production in Beijin-g. Gansu Agr. Sci. and Techn. 1, 81-87 (2018)

7. X. Yang , Y. Y Mu and X D. Wang .Vegetable Produc-tion Characteristics in Beijing.Chinese Sci Bulletin, 32(13), 182-190 (2016)

8. Fan S. X. Study on the development of leaf vegetable indus-try in Beijing. China Agriculture Press (2014)

9. S L. Cui. Identification and prevention of common diseases of lettuce. China Fruit \& Vegetable, 6, 35-35 (2007)

10. Miloševic' N, Sekulic'P and Cvijanovic' G. Microorganisms 
as bioindicators of pollutants in soil. Ratarstvo $I$ Povrtarstvo (2010)

11. Isabel, D, Raymond, G and Malcolm, L. Soil microbial community response to land-management and depth, related to the de-gradation of organic matter in English wetlands:implications for the in situ preservation of archaeological remains. Appl Soil Ecol,44(3), 219-227 (2010)

12. J L. Hu and X G. Lin. Soil microorganisms and soil health: biological indicators and ecological regulation. The seventh national symposium on soil biology and biochemistry and the second national symposium on soil health (2014)

13. P. Wang, B. Chen andH. Zhang. High throughput sequencin-g analysis of bacterial communities in soils of a typical Poyang Lake wetland. Acta Ecologica Sinica, 37(5),1650-1658 (2017)

14. Amato, K. R, Yeoman, C. J and Kent, A, et al. Habitat degr-adation impacts black howler monkey(alouatta pigra)gastrointestinalmicrobiomes. ISME J, 7(7), 1344-1353 (2013)

15. W J. Staddon, L C. Duchesne and J T. Trevors. Microbial Diversity and Community Structure of Postdisturbance Forest Soils as Determined by Sole-Carbon-Source Utilization Patterns. Micro-b Ecol, 34(2):125-130 (1997)

16. F. Zhao, M Z. Zhao and Y. Wang, et al. Biodiversity of ba-cteria and fungi in rhizosphere of strawberry with different conti-nuous cropping years. Microbiology,44(6), 1377-1386 (2017)

17. X. Ouyang, X L. Ruan and C. Wu, et al. Main bacterial groups in banana soil under rotated and continuous cropping. J APPL ECOL, 22(6),1573-1578 (2011)

18. J. hao,T. Ni and J. Li, et al. Effects of organic-inorganic compound fertilizer with reduced chemical fertilizer application oncrop yields, soil biological activity and bacterial community struc-ture in a rice-wheat cropping system. Appl Soil Ecol, 99(18), 1-12 (2016)

19. C. Liu ,Y. Huang and X. Chen, et al. Effects of Proporti-on of Organic and Inorganic Fertilizers on Tobacco-planting Soil Bacteria Composition, Yield and Quality of Flue-cured Tobacco. J AGR SCI,47(6), 58-64 (2018)

20. L L.Chen, J. Liu and M D. Li , et al. Diversity of soil ba-cteria under different tillage methods. J MICROBIO-L, 38(4), 68-76 (2018)

21. Q. Li , L. Yuan and S P.Yang, et al. Influence of continu-ous cropping on growth of Artemisia annua and bacterial commu-nities in soil. Chinese Materia Medica, 41(10),1803-1810 (2016)

22. J. Aislabie, D J.Saul and J M.Foght . Bioremediation of hydrocarbon-contaminated polarsoils. Extremophiles, 10(3):171-179 (2006)

23. Liu, X, Zhang, J and Gu, T, et al. Microbial community diversities and taxa abundances in soils along a seven-year gradi-ent of potato monoculture using high throughput pyrosequencing approach. PLOS ONE,9(1)（2014）

24. R P. Yang, Y L. Mo and C M. Liu , et al. The effects of cattle manure and garlic rotation on soil under continuous croppin-g of watermelon (Citrullus lanatus L.). PLOS ONE, 11(6)（2016）

25. W. Xiong, Q Y. Zhao and C. Xue, et al. Comparison of fungal community in black pepper-vanilla and vanilla monoculture systems associated with vanilla fusarium wilt disease. Front Micr-obiol, 9(7),117. (2016)

26. Y Y. Ma ,Y L. Li and H X. Lai, et al. Effect of sick rhiz-osphere soil under tomato continuous cropping onsoil nematodes, microbes and tomato growth. China. J. EcoAgric. 25(5), 730-739 (2017) 